\title{
Nuclear medicine practice in the field of infection and inflammation imaging: a pragmatical survey
}

\author{
Salvatore Annunziata ${ }^{1} \cdot$ Giorgio Treglia ${ }^{2,3,4} \cdot$ Francois Jamar $^{5} \cdot$ Chiara Lauri $^{6} \cdot$ Christopher J. Palestro $^{7}$. \\ Olivier Gheysens ${ }^{5} \cdot$ Andor W. J. M. Glaudemans $^{8}$
}

Published online: 17 February 2022

(c) The Author(s), under exclusive licence to Springer-Verlag GmbH Germany, part of Springer Nature 2022

During the last decade, the interest in nuclear medicine techniques for diagnosing infections has been growing [1]. There is an increasing body of evidence to support the use of $\left[{ }^{18} \mathrm{~F}\right]-\mathrm{FDG}$ positron emission tomography/computed tomography $\left(\left[{ }^{18} \mathrm{~F}\right]\right.$-FDG-PET/CT $)$ for several indications in this field. Nevertheless, $\left[{ }^{18} \mathrm{~F}\right]$-FDG has some important limitations and pitfalls, especially in patients with foreign body material [2]. In these cases, differentiation between reactive inflammation and infection can be challenging and a correct diagnosis is of utmost importance for subsequent treatment. For these cases, white blood cell (WBC) scintigraphy is a highly valuable technique that allows for the visualization of the dynamics of white blood cells, hence differentiation between sterile inflammation and infection with high accuracy [3].

Back in 2013, a first general joint EANM/SNM guideline about the use of $\left[{ }^{18} \mathrm{~F}\right]$-FDG-PET/CT for inflammation and infection was published [4]. This guideline was based on limited data available at that time, and the authors claimed that this guideline could not be seen as definitive given the rapidly evolving use of $\left[{ }^{18} \mathrm{~F}\right]$-FDG-PET/CT. Now, 9 years

This article is part of the Topical Collection on Infection and inflammation

Andor W. J. M. Glaudemans

a.w.j.m.glaudemans@umcg.nl

1 Unità Di Medicina Nucleare, TracerGLab, Dipartimento Diagnostica Per Immagini, Radioterapia Oncologica ed Ematologia, Fondazione Policlinico A. Gemelli IRCCS, Largo Agostino Gemelli 8, 00168 Rome, Italy

2 Clinic of Nuclear Medicine, Imaging Institute of Southern Switzerland, Ente Ospedaliero Cantonale, Bellinzona, Switzerland

3 Faculty of Biomedical Sciences, Università della Svizzera italiana, Lugano, Switzerland

4 Faculty of Biology and Medicine, University of Lausanne, Lausanne, Switzerland later, we agree with this statement, since not only there is accumulating evidence for existing indications, but also more and more data are available in new indications, and in larger patient populations. Several important EANM guidelines were developed for the correct use of $\left[{ }^{18} \mathrm{~F}\right]$-FDG-PET/ $\mathrm{CT}$ and WBC scintigraphy in infectious diseases [3,5-11]. These guidelines are evidence-based, and include the correct patient preparation, the correct image acquisition, and the correct analysis and interpretation of the scans. Moreover, NM techniques have been incorporated in several clinical guidelines, written in collaboration with clinical societies, in which the preferred imaging technique, the correct use of the technique, and the time point in the disease course when the technique should be used are described and illustrated by diagnostic flowcharts, e.g. for endocarditis [6, 7], vascular graft infections [8], peripheral bone infection in adults [9], prosthetic joint infection [10], and spondylodiscitis [11].

To evaluate the use of these guidelines in clinical practice, a survey was performed to collect information on the use of $\left[{ }^{18} \mathrm{~F}\right]$-FDG-PET/CT and WBC scintigraphy for infectious/inflammatory disorders. The goal of this survey was to provide an answer to the following questions: Are all centres equipped to perform these imaging modalities? Do they use these imaging techniques and if yes, how often and for which

5 Department of Nuclear Medicine, Cliniques Universitaires Saint-Luc and Institute of Clinical and Experimental Research (IREC), Université Catholique de Louvain (UCLouvain), Avenue Hippocrate 10, B-1200 Brussels, Belgium

6 Nuclear Medicine Unit, Department of Medical-Surgical Sciences and of Translational Medicine, Faculty of Medicine and Psychology, Sapienza" University of Rome, Rome, Italy

7 Donald and Barbara Zucker School of Medicine, Hofstra University and Northwell Health, Hempstead, NY, USA

8 Medical Imaging Center, Department of Nuclear Medicine and Molecular Imaging, University of Groningen, University Medical Center Groningen, Hanzeplein 1, 9700, RB, Groningen, the Netherlands 
indications? Do they use these techniques in the correct way, standardized to guidelines? Which guidelines do they use? And which technique do they prefer for which indication and is this in concordance with the guidelines?

A web-questionnaire was prepared by the authors to evaluate concordances and discrepancies in daily NM practice in Europe in the field of infection imaging, in line with recent recommendations and requirements to maximize response rates such as a personal introductory statement; the offer to make results public; the use of simple headers and textual representation of response categories; and the prerequisite of a relatively short deadline including several reminders $[12,13]$. The questionnaire was available in a Google Form Document. As of March 1, 2021, an invitation to the survey was sent to the corresponding authors of scientific articles in the field of NM infection imaging indexed in SCOPUS in 2020, using the following query string: ("infection") AND ("nuclear medicine" or "pet" or "positron" or "scintigraphy" or "spet" or "spect"). Two reminders were sent to all non-responding recipients. To further increase potential responses, the survey weblink was accessible by social media.

Fifty questions in English were prepared for NM professionals dedicated to infection imaging worldwide. Question types were dichotomous, single-choice, multiple-choice, rating scales, or open-ended for number, text comment, or date [12, 13]. General questions about personal and demographic data were included. Subsequently, specific questions to evaluate concordances and discrepancies in daily $\mathrm{NM}$ practice in Europe in the field of infection imaging were prepared to address the following main issues: (1) general demographic, hospital and NM department information; (2) $\left[{ }^{18} \mathrm{~F}\right]$-FDG-PET/CT protocols; (3) WBC protocols; (4) clinical indications; and (5) reimbursement. All data about demographics, hospital, department, protocols, indications, and reimbursement were analyzed per individual responder.

In total, 101 individual responses were received, 92 from specialists working in Europe and (only) 9 from specialists working in North America. Given the low number of nonEuropean responders, quantitative analysis focused on the 92 individual responses from Europe. Nearly all responders were from NM departments: only a few responders were from Radiology departments. Most of the responders were NM physicians $(84 / 92,92 \%)$ working in an academic hospital (50/92, 54\%) (Fig. 1).

Most departments were equipped with both PET/CT and SPECT/CT (73/92, 80\%), mostly using both $\left[{ }^{18} \mathrm{~F}\right]-\mathrm{FDG}$ and HMPAO-labelled WBCs for infection imaging, respectively. Many responders $(39 / 92,42 \%)$ performed less than five PET scans weekly and the vast majority $(72 / 92,78 \%)$ performed less than five SPECT scans weekly for infectious indications. The three main indications for infection imaging were
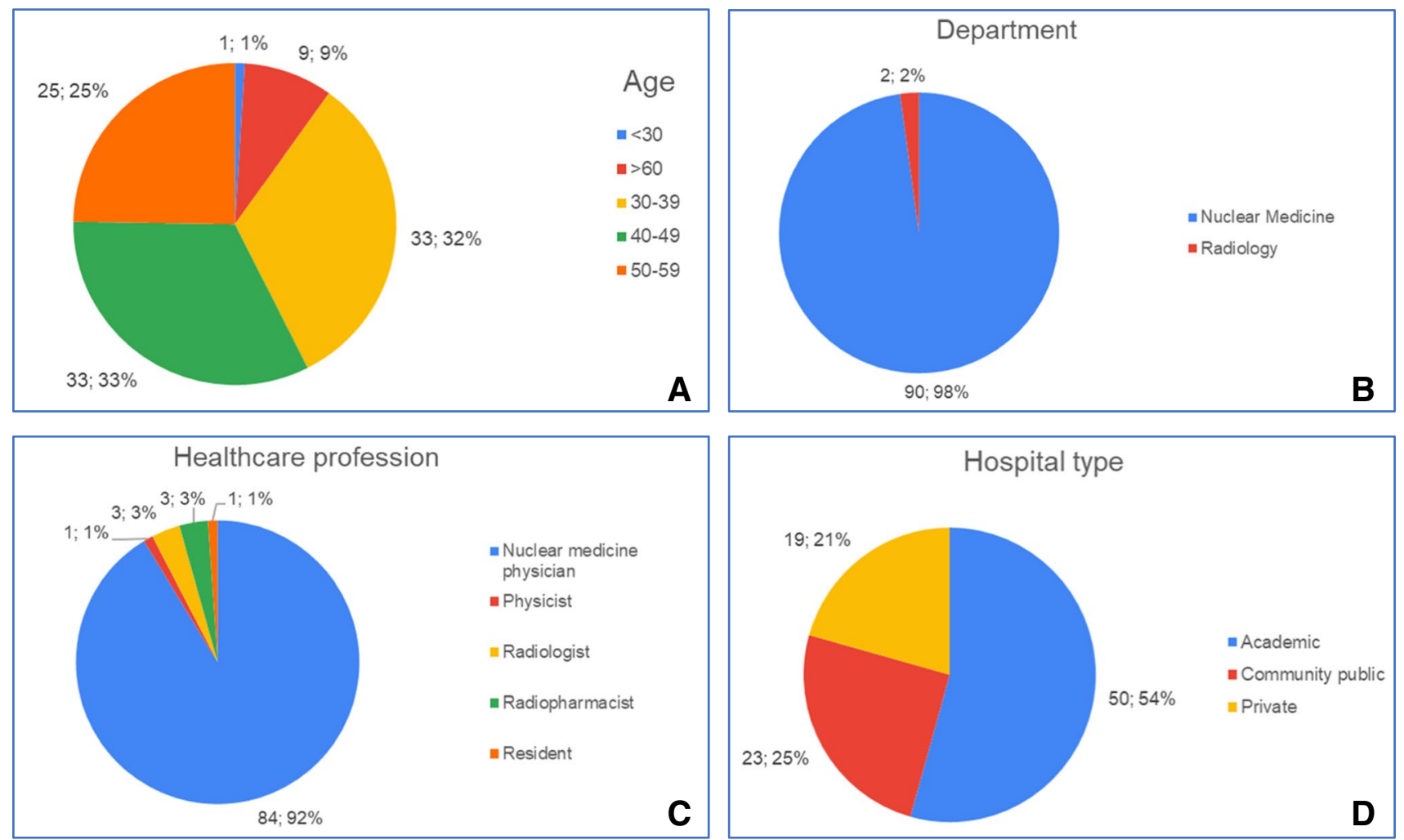

Fig. 1 General demographic and hospital information, such as age (a), department (b), healthcare profession (c), and hospital type (d) 
FUO, sepsis/bacteraemia, and endocarditis/CIED, followed by vascular graft infection and prosthetic joint infection. For infection imaging, most of the responders had their own institutional protocols $(74 / 92,80 \%)$, and followed EANM guidelines in most centres $(70 / 92,76 \%)$, either stand-alone EANM in 53\%, or in combination with clinical guidelines in $23 \%$ (Fig. 2).

Most responders used $3 \mathrm{MBq} / \mathrm{kg}(41 / 92,45 \%)$ for $\left[{ }^{18} \mathrm{~F}\right]$-FDG-PET and 6-h fasting duration for infection imaging (60/92, 65\%). A significant part of the responders (26/92, $28 \%$ ) used a 12-h fasting duration for infection PET scans. A specific diet was in most centres suggested only for endocarditis and CIED (65/92, 71\%), mostly consisting of a highfat low carbohydrate diet with or without prolonged fasting or heparin administration. Fifteen responders (16\%) used a specific diet for all infectious indications; twelve responders (13\%) used no specific diet at all. A glycaemia cut-off of $200 \mathrm{mg} / \mathrm{dl}(68 / 92,74 \%)$ and scan time point at $60 \mathrm{~min}$ after injection $(80 / 92,87 \%)$ were largely used (Fig. 3).

In total, 69 of the 92 responders used WBC imaging. ${ }^{99 \mathrm{~m}} \mathrm{Tc}-\mathrm{HMPAO}$ alone was the most used tracer for WBC imaging in our cohort (40/69,58\%), with an injected activity ranging between 185 and $550 \mathrm{MBq}$. The remaining centres used ${ }^{99 \mathrm{~m}} \mathrm{Tc}$-antigranulocyte monoclonal antibodies (14/62, $23 \%$ ), a combination of ${ }^{99 \mathrm{~m}} \mathrm{Tc}-\mathrm{HMPAO}$ and ${ }^{99 \mathrm{~m}} \mathrm{Tc}$-antigranulocyte monoclonal antibodies $(9 / 62,15 \%)$, a combination of ${ }^{99 \mathrm{~m}} \mathrm{Tc}-\mathrm{HMPAO}$ and ${ }^{111} \mathrm{In}$-oxine labelled WBC $(2 / 62,3 \%)$, or a combination of all three possibilities $(3 / 62,5 \%)$. Only one centre used only ${ }^{111} \mathrm{In}$-oxine for WBC labelling. The number of scans performed for ${ }^{99 \mathrm{~m}} \mathrm{Tc}-\mathrm{HMPAO} \mathrm{WBC}$ imaging differed. Most of them used two imaging time points (at 2-4 h and 20-24 h) in 32 out of 60 responses (53\%), or three imaging time points (including a scan at $30 \mathrm{~min}$ $(18 / 60,30 \%)$. Other centres used only 1 imaging time point $(3 / 60,5 \%)$ or more than 3 imaging time points $(7 / 60,12 \%)$. Regarding the time points of these scan sessions, there was a lot of difference, with scans performed after $30 \mathrm{~min}$, between 2 and $4 \mathrm{~h}$, between $4-8 \mathrm{~h}$, and between 20 and $24 \mathrm{~h}$. Both planar and SPECT imaging are mostly considered (53/67, $79 \%$ ), with just in 34 out of 67 (51\%) using an acquisition time corrected for ${ }^{99 \mathrm{~m}} \mathrm{Tc}$ decay. Most of the responders used EANM procedural guidelines for WBC infection imaging (alone or with other ones), but not all centres applied the recommended scan time points, and used decay-corrected acquisition times (Fig. 4).

Participants were asked about which imaging modality they performed first for the main clinical infectious conditions. $\left[{ }^{18} \mathrm{~F}\right]$-FDG-PET/low-dose CT was largely used as first imaging modality in adults $(57 / 92,62 \%)$ and children (74/92, 81\%) with FUO, prosthetic (54/92, 59\%) and native $(48 / 92,52 \%)$ valve endocarditis, myocardial inflammation $(60 / 92,65 \%)$, vascular graft infection $(47 / 92,51 \%)$, and spondylodiscitis $(50 / 92,54 \%)$. For these indications, ${ }^{99 \mathrm{~m}} \mathrm{Tc}$ HMPAO-labelled WBC was rarely used, except for vascular
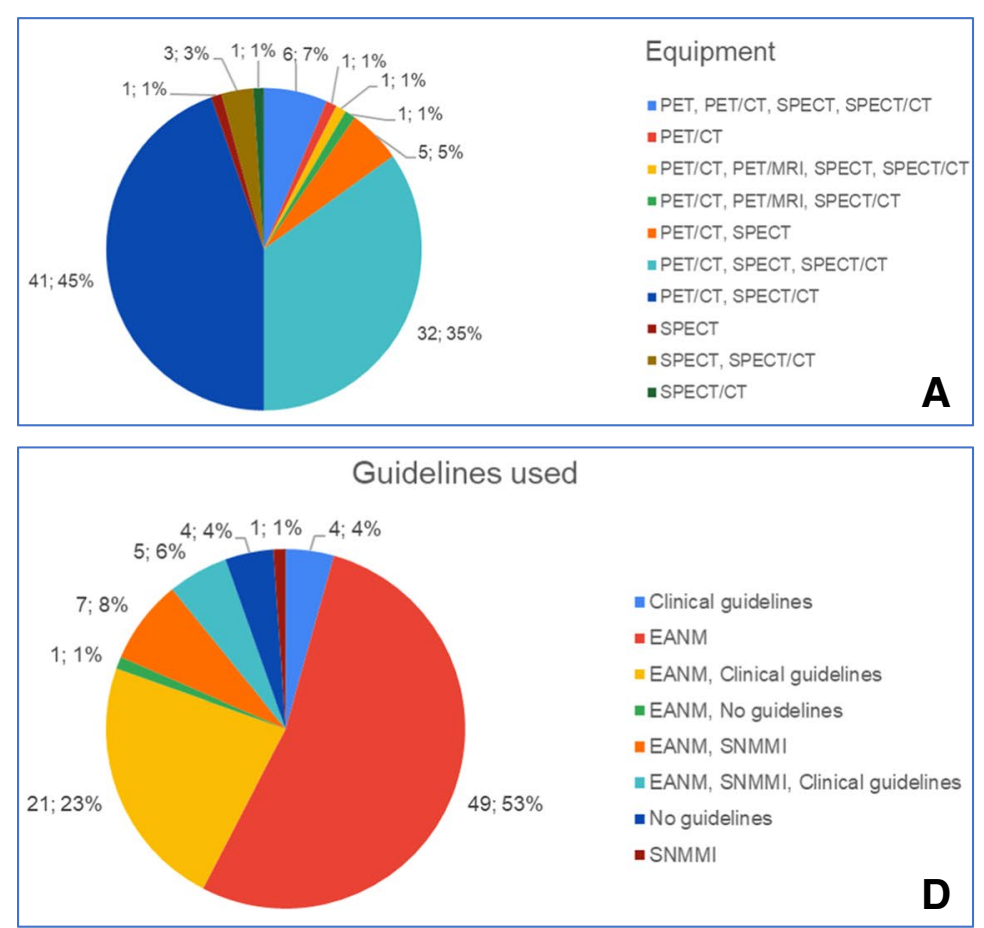

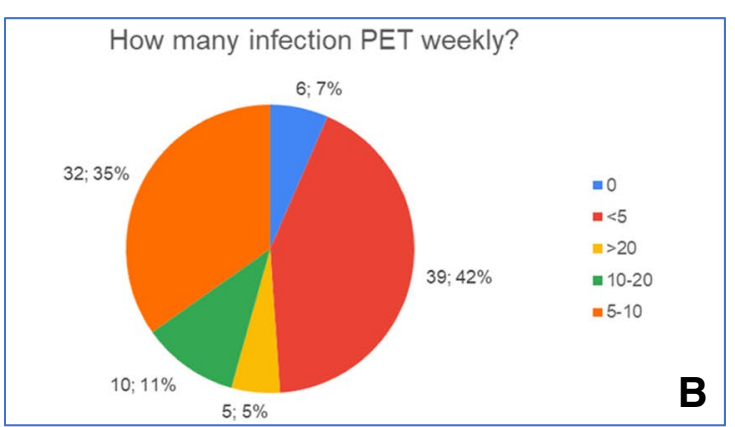

How many infection SPECT weekly?

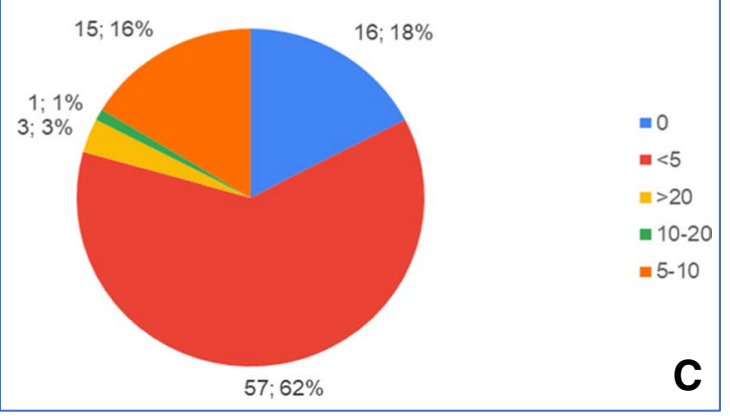

Fig. 2 Data about general NM department information on infection imaging, such as equipment (a), number of PET (b) and SPECT scans (c), and guidelines used (d) 

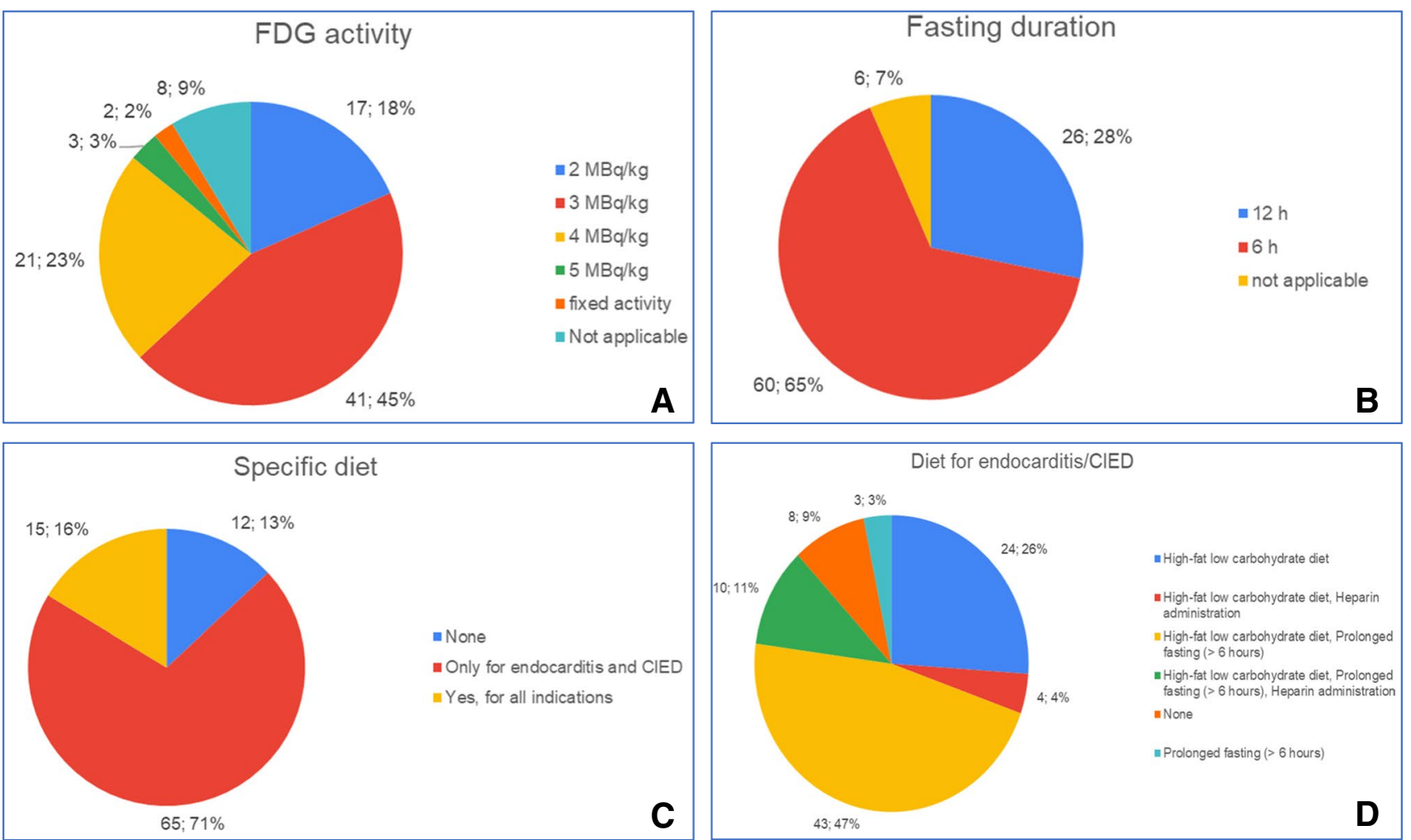

Fig. 3 Data about $\left[{ }^{18} \mathrm{~F}\right]$-FDG-PET protocols, in terms of administered activity (a), fasting duration (b), specific diet (c), and diet for endocarditis (d)
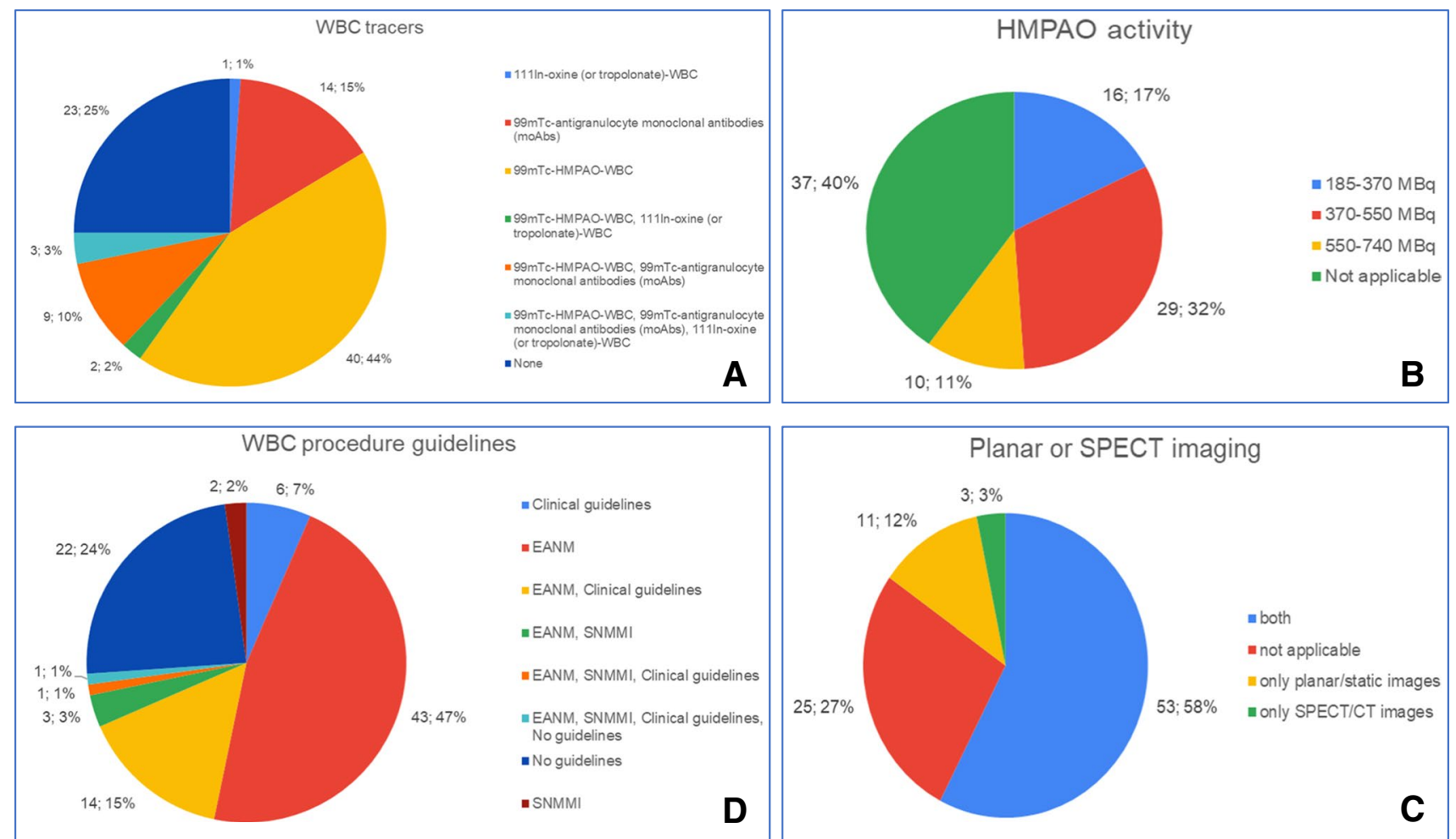

Fig. 4 Data about WBC protocols, such as tracers used (a), activity for HMPAO (b), planar/SPECT imaging (c), and WBC procedure guidelines (d) 
graft infection (16/92, 17\%). In some centres, ${ }^{67} \mathrm{Ga}$ or ${ }^{68} \mathrm{Ga}-$ citrate was used as first imaging modality. ${ }^{99 \mathrm{~m}} \mathrm{Tc}-\mathrm{HMPAO}$ WBC scintigraphy was mainly used as first imaging modality in suspected prosthetic joint infections and in fracturerelated infections (with and without implants), which is in accordance with EANM diagnostic flowcharts (Fig. 5).

Hospital and physician reimbursement for infection imaging did not differ amongst responders, with a prevalent absence of specific infection imaging costs. Prevalent reimbursement schemes were between 200 and 500 euros for ${ }^{99 \mathrm{~m}} \mathrm{Tc}-\mathrm{HMPAO}$ WBC scintigraphy and between 500 and 1000 euros for FDG-PET/low-dose CT, respectively.

We were happy to learn that the majority used institutional protocols and followed existing guidelines. However, the protocols were not always implemented according to the recommendations, especially not in WBC scintigraphy. The three main indications for infection imaging were FUO, sepsis/bacteraemia, and endocarditis/CIED. $\left[{ }^{18} \mathrm{~F}\right]$-FDG-PET/ low-dose CT was largely used as first imaging modality in adults and children with FUO, prosthetic and native valve endocarditis, myocardial inflammation, vascular graft infection, and spondylodiscitis. In contrast, ${ }^{99 \mathrm{~m}} \mathrm{Tc}-\mathrm{HMPAO} \mathrm{WBC}$ scan was mainly used as first imaging modality in fracturerelated infections with and without implants and in prosthetic joint infections.
In general, the overall number of PET and SPECT scans performed for infections was quite low, in $77 \%$ less than ten scans per week. Only a limited number of departments perform 10-20 PET scans for infection per week (11\%), and in only $5 \%$ of the departments more than 20 PET scans were performed weekly. For SPECT scans, the results are less than 10 scans per week in $78 \%$ of the responses, in 1\% 10-20 scans per week, and in $3 \%$ more than 20 scans per week. Remarkably, $18 \%$ and $7 \%$ of the responders do not perform or has no access to SPECT or PET scans, respectively. These differences could be explained by the variable availability and reimbursement and to the fact that not all referring clinicians do request a nuclear medicine scan, despite the existing evidence. Therefore, it is important to have joint clinical guidelines and diagnostic algorithms, to widely adopt NM techniques in every centre based on existing evidence and not only by clinicians who believe in and/or are familiar with NM techniques.

Looking particular into the protocols used for $\left[{ }^{18} \mathrm{~F}\right]-\mathrm{FDG}-\mathrm{PET} / \mathrm{CT}$, the majority used an administered $\left[{ }^{18} \mathrm{~F}\right]-\mathrm{FDG}$ activity of $3 \mathrm{MBq} / \mathrm{kg}(45 \%)$, but $26 \%$ administer 4 or $5 \mathrm{MBq} / \mathrm{kg}$ which should be decreased for the benefit of both the technologists and patients, for radiation protection issues. On the contrary, in $18 \%$ of cases, $2 \mathrm{MBq} / \mathrm{kg}$ is injected into the patients. This is most likely
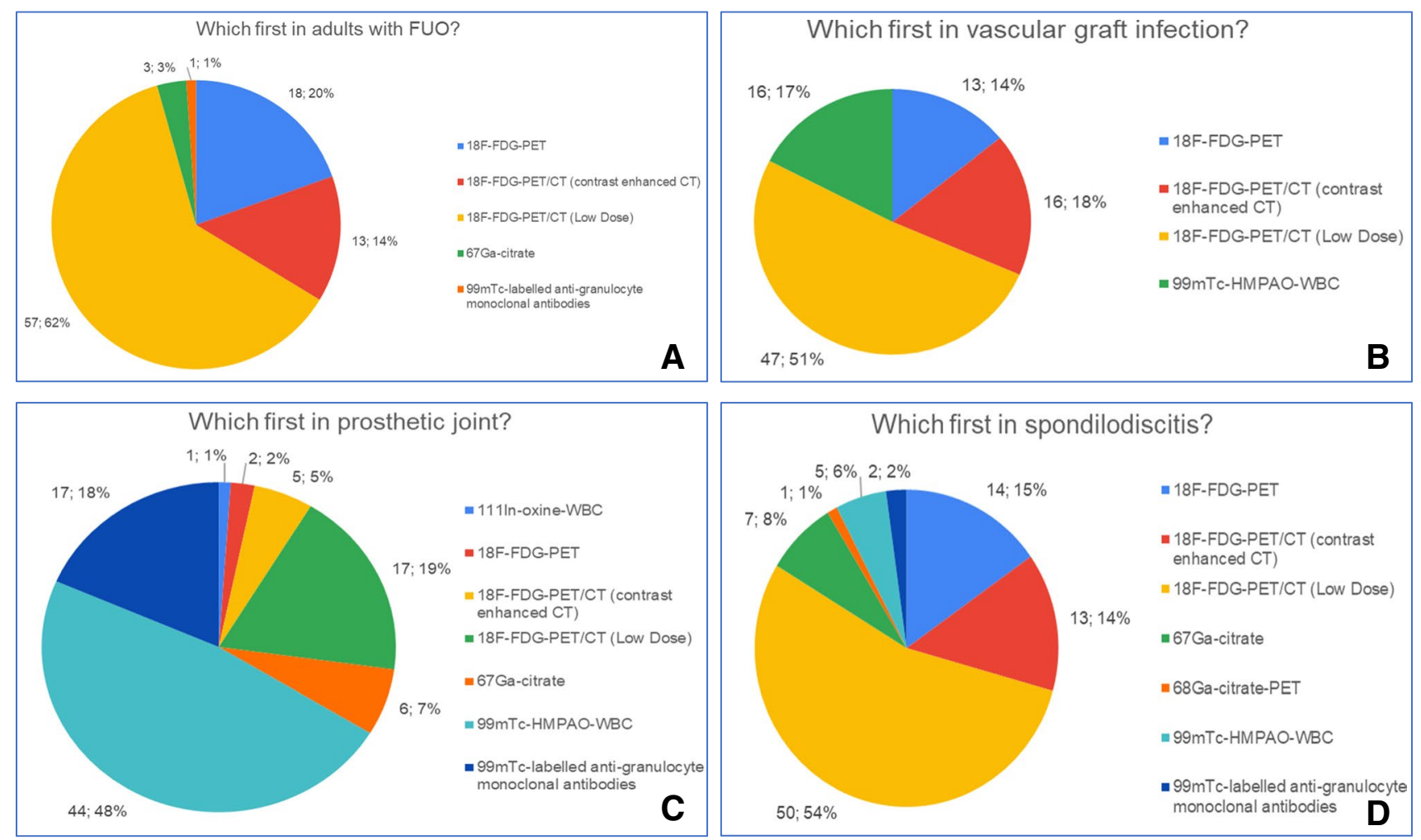

Fig. 5 Data about clinical indications, in terms of which imaging modality performed first in the following scenarios: adults with FUO (a), vascular graft infection (b), prosthetic joint infection (c), and spondylodiscitis (d) 
attributed to the newer (digital) PET/CT camera systems that are used more frequently and administered activities should be adapted to the existing equipment. We also observed differences in the specific diet used, not only between the indications (only for endocarditis or for all indications), but also between the diets used (no diet at all, high-fat low carbohydrate with or without prolonged fasting and with or without heparin). This could be more standardized between users according to a recently published recommendation paper [5].

For WBC scintigraphy, marked differences were found in the tracers used $\left({ }^{99 \mathrm{~m}} \mathrm{Tc}-\mathrm{HMPAO},{ }^{111} \mathrm{In}\right.$-oxine, or ${ }^{99 \mathrm{~m}} \mathrm{Tc}$ antigranulocyte monoclonal antibodies), in administered activity, in scanning time points, and in scan acquisition with or without decay correction. In a recent EANM guideline (3), and in several studies including homogenous and large patient populations [14, 15], it was demonstrated that scanning times corrected for decay yielded a better diagnostic accuracy. However, this was only used in 51\% of the respondents using WBC scintigraphy. This leaves room for optimization and standardization amongst centres, to avoid misinterpretation, and, as a consequence, a lower diagnostic accuracy compared to scanning times corrected for decay.

Despite several efforts, only nine responses outside Europe were received. The reason for this limited response rate remains unclear, but one of the explanations could be the lack of reimbursement by the CNS of $\left[{ }^{18} \mathrm{~F}\right]$-FDG-PET for infectious indications until recently, so probably only a few centres use $\left[{ }^{18} \mathrm{~F}\right]$-FDG-PET/CT for these indications. Finally, the time has come for the USA to embrace $\left[{ }^{18} \mathrm{~F}\right]-\mathrm{FDG}$ as the molecular imaging test of choice for many inflammatory and infectious indications [16]. Further research should focus on standardization and harmonization worldwide in order to achieve the same and optimal clinical practice for patients with infections complications everywhere.

In summary, this survey showed that there is a large consensus on the preferred nuclear medicine imaging technique for specific infectious indications and the adherence to the existing EANM/joint clinical guidelines to correctly use $\left[{ }^{18} \mathrm{~F}\right]$-FDG-PET/CT and WBC scintigraphy. Nevertheless, efforts should be made to have the techniques available everywhere, to adhere to the guidelines in every centre, and to interact with the clinicians to adopt our techniques based on the available evidence and existing guidelines. Furthermore, a revision of the 2013 evidence-based guideline for the use of $\left[{ }^{18} \mathrm{~F}\right]$-FDG-PET/CT in infection and inflammation is highly needed, since new indications, new patient data, and new guidelines have become available.

Acknowledgements We are grateful to all the responders of this survey for their participation and support.

\section{Declarations}

Ethics approval All procedures performed in studies involving human participants were in accordance with the ethical standards of the institutional and/or national research committee and with the 1964 Helsinki Declaration and its later amendments or comparable ethical standards.

Informed consent Informed consent to anonymously collect and publish personal and opinion data of this survey was obtained in the presentation page of the web questionnaire.

Conflict of interest The authors declare no competing interests.

\section{References}

1. Treglia G. Diagnostic performance of ${ }^{18} \mathrm{~F}-\mathrm{FDG}$ PET/CT in infectious and inflammatory diseases according to published metaanalyses. Contrast Media Mol Imaging. 2019;2019:3018349.

2. Pijl JP, Nienhuis PH, Kwee TC, Glaudemans AWJM, Slart RHJA, Gormsen LC. Limitations and pitfalls of FDG-PET/CT in infection and inflammation. Semin Nucl Med. 2021;51(6):633-45.

3. Signore A, Jamar F, Israel O, Buscombe J, Martin-Comin J, Lazzeri E. Clinical indications, image acquisition and data interpretation for white blood cells and anti-granulocyte monoclonal antibody scintigraphy: an EANM procedural guideline. Eur J Nucl Med Mol Imaging. 2018;45(10):1816-31.

4. Jamar F, Buscombe J, Chiti A, Christian PE, Delbeke D, Donohoe $\mathrm{KJ}$, et al. EANM/SNMMI guideline for 18F-FDG use in inflammation and infection. J Nucl Med. 2013;54(4):647-58.

5. Slart RHJA, Glaudemans AWJM, Gheysens O, Lubberink M, Kero T, Dweck MR, et al. 4Is Cardiovascular imaging: a joint initiative of the European Association of Cardiovascular Imaging (EACVI); European Association of Nuclear Medicine (EANM). Procedural recommendations of cardiac PET/CT imaging: standardization in inflammatory-, infective-, infiltrative-, and innervation (4Is)-related cardiovascular diseases: a joint collaboration of the EACVI and the EANM. Eur J Nucl Med Mol Imaging. 2021;48(4):1016-39.

6. Habib G, Lancellotti P, Antunes MJ, Bongiorni MG, Casalta JP, Del Zotti F, et al. ESC Scientific Document Group. 2015 ESC Guidelines for the management of infective endocarditis: The Task Force for the Management of Infective Endocarditis of the European Society of Cardiology (ESC). Endorsed by: European Association for Cardio-Thoracic Surgery (EACTS), the European Association of Nuclear Medicine (EANM). Eur Heart J. 2015;36(44):3075-128.

7. Erba PA, Pizzi MN, Roque A, Salaun E, Lancellotti P, Tornos P, Habib G. Multimodality imaging in infective endocarditis: an imaging team within the endocarditis team. Circulation. 2019;140(21):1753-65.

8. Chakfé N, Diener H, Lejay A, Assadian O, Berard X, Caillon J, et al. Editor's Choice - European Society for Vascular Surgery (ESVS) 2020 Clinical practice guidelines on the management of vascular graft and endograft infections. Eur J Vasc Endovasc Surg. 2020;59(3):339-84.

9. Glaudemans AWJM, Jutte PC, Cataldo MA, Cassar-Pullicino V, Gheysens O, Borens O, et al. Consensus document for the diagnosis of peripheral bone infection in adults: a joint paper by the EANM, EBJIS, and ESR (with ESCMID endorsement). Eur J Nucl Med Mol Imaging. 2019;46(4):957-70.

10. Signore A, Sconfienza LM, Borens O, Glaudemans AWJM, Cassar-Pullicino V, Trampuz A, et al. Consensus document for the diagnosis of prosthetic joint infections: a joint paper by the 
EANM, EBJIS, and ESR (with ESCMID endorsement). Eur J Nucl Med Mol Imaging. 2019;46(4):971-88.

11. Lazzeri E, Bozzao A, Cataldo MA, Petrosillo N, Manfrè L, Trampuz A, et al. Joint EANM/ESNR and ESCMID-endorsed consensus document for the diagnosis of spine infection (spondylodiscitis) in adults. Eur J Nucl Med Mol Imaging. 2019;46(12):2464-87.

12. Annunziata S, Bauckneht M, Albano D, Argiroffi G, Calabrò D, Abenavoli E, et al. Impact of the COVID-19 pandemic in nuclear medicine departments: preliminary report of the first international survey. Eur J Nucl Med Mol Imaging. 2020;47(9):2090-9.

13. Annunziata S, Albano D, Laudicella R, Bauckneht M. Young Committee of the Italian Association of Nuclear Medicine (AIMN). Surveys on COVID-19 in nuclear medicine: what happened and what we learned. Clin Transl Imaging. 2020;24:1-3.

14. Glaudemans AW, de Vries EF, Vermeulen LE, Slart RH, Dierckx RA, Signore A. A large retrospective single-centre study to define the best image acquisition protocols and interpretation criteria for white blood cell scintigraphy with ${ }^{99} \mathrm{mTc}-\mathrm{HMPAO}-$ labelled leucocytes in musculoskeletal infections. Eur J Nucl Med Mol Imaging. 2013;40(11):1760-9.

15. Erba PA, Leo G, Sollini M, Tascini C, Boni R, Berchiolli RN, et al. Radiolabelled leucocyte scintigraphy versus conventional radiological imaging for the management of late, low-grade vascular prosthesis infections. Eur J Nucl Med Mol Imaging. 2014;41(2):357-68.

16. Wahl RL, Dilsizian V, Palestro CJ. At Last, ${ }^{18}$ F-FDG for inflammation and infection! J Nucl Med. 2021;62(8):1048-9.

Publisher's note Springer Nature remains neutral with regard to jurisdictional claims in published maps and institutional affiliations. 